Revista Mexicana de Economía y Finanzas Nueva Época

Volumen 14 Número 4, Octubre - Diciembre 2019, pp. 729-743

DOI: https://doi.org/10.21919/remef.v14i4.107

\title{
Impacto de la rentabilidad y la cuota de mercado en las empresas zombis en México
}

\author{
Manuel Humberto De la Garza Cárdenas \\ Universidad Autónoma de Tamaulipas, México \\ Yesenia Sánchez Tovar \\ Universidad Autónoma de Tamaulipas, México \\ Mariana Zerón Félix \\ Universidad Autónoma de Tamaulipas, México
}

(Recepción: 18/noviembre/2018, aceptado: 28/marzo/2019)

\section{Resumen}

El objetivo de este trabajo consiste en profundizar en la presencia de empresas zombis en la Bolsa Mexicana de Valores (BMV) y los factores que inciden en su existencia. Para ello, a través del método CHK se determinó la presencia de empresas zombis en México y, posteriormente, mediante un modelo logit se evalúa el impacto de la rentabilidad y la cuota de mercado en las empresas zombi. Los datos empleados corresponden a 102 empresas inscritas en el mercado de valores de México en el año 2015, lo cual permitió identificar la presencia de 18 empresas con características zombis. Mientras que el análisis empírico confirmó que la cuota de mercado impacta positivamente a las empresas zombis, mientras que la rentabilidad disminuye la probabilidad de incurrir en esta situación. El modelo empleado representa uno de los trabajos pioneros en América Latina sobre este tipo de empresas y da luz sobre el comportamiento que siguen dichas organizaciones mostrando que las características del mercado pueden ser otros predictores importantes de las empresas zombis y representan futuras líneas de investigación relevantes.

Clasificación JEL: G33, G32, M10

Palabras clave: empresas zombis, competitividad empresarial, costo de deuda

\section{Identification of zombie companies in Mexico, impact of profitability and market share in them}

\section{Abstract}

The objective of this paper is to deepen the presence of zombie companies in the Bolsa Mexicana de Valores (BMV) and the factors that affect its existence. To do this, through the CHK method, the presence of zombie companies in Mexico was determined and, subsequently, through a logistic model, the impact of profitability and market share on zombie companies was evaluated. The data correspond to 102 firms registered in the BMV in 2015, in which the presence of 18 companies with zombie characteristics will be identified. While empirical analysis confirms that market share positively impacts zombie companies, while profitability decreases the probability of present in this situation. The model used represents one of the pioneering works in Latin America this type of companies and the light on the behavior that they follow as the samples and the characteristics of the market can be other important predictors the zombie companies and the corresponding relevant research lines.

JEL Classification: G33, G32, M10

Keywords: zombie firms, business competitiveness, cost of debt 


\section{Introducción}

Éste trabajo busca contribuir al entendimiento de las empresas zombis, particularmente en los mercados Latinoamericanos, por ello, como punto de partida se debe comprender el término "zombi" en el ámbito académico, destacando las características de las firmas que se clasifican como tales.

El uso de éste adjetivo en las ciencias sociales es reciente y poco común; se refiere a un conjunto de características particulares que permite la categorización de una condición (Hoshi, 2006). Fue en Japón, durante la década de 1990 cuando se identificaron por primera vez éste tipo de empresas, al encontrar firmas poco competitivas y con resultados financieros negativos que permanecieron activas en los distintos mercados pese a que naturalmente deberían de haber cerrado (Amato \& Fantacci, 2016; Camacho, Segovia, \& Pascual, 2015; Ito \& Sasaki, 2002; Uchida et al., 2015). Sin embargo, durante el nuevo milenio se encontraron este tipo de empresas en distintos países del mundo dadas las prácticas empresariales similares que son empleadas en las distintas economías (McGowan, Andrews, \& Millot, 2016).

Esta condición adquirida por las empresas es resultado de las decisiones de la administración, ya que es ella la que solicita los recursos financieros y dicta el destino del mismo, así como también la cantidad y la forma de realizar las actividades productivas (Tan, Huang, \& Woo, 2016). En éste sentido se denota que, generalmente, las empresas zombis requieren el financiamiento al presentan bajos niveles de productividad y de ventas (McGowan et al., 2016). Lo anterior refleja, además de los resultados financieros y operativos negativos, en una baja presencia en el mercado o mercados altamente concentrados, es decir, industrias con características oligopólicas (Hoshi, 2006; Imai, 2016; Iwaisako, Fukuoka, \& Kanou, 2013). En cuanto a la fuerza laboral, éstas firmas cuentan con un número estable de trabajadores puesto que buscan evitar incurrir en costos, tanto de contratación como de liquidación (Caballero, Hoshi, \& Kashyap, 2008).

Como se mencionó anteriormente, la empresa zombi, no es una condición exclusiva de los países asiáticos, encontrándose esta característica en países como China, España y miembros de la OCDE (McGowan et al., 2016; Tan et al., 2016; Urionabarrenetxea, Garcia-Merino, San-Jose, \& Retolaza, 2018), por lo anterior el presente artículo tiene como objetivo identificar la existencia de empresas zombis en México y determinar si el rendimiento y la cuota de mercado predice este comportamiento. Para la consecución de dichos objetivos, el trabajo se estructuró de la siguiente forma, posterior a la introducción se explora la definición de éste tipo de empresas. Se continúa con un apartado sobre los predictores de este tipo de empresas, así como una revisión de estudios empíricos que evalúan las relaciones planteadas; se continua con la exposición de la unidad de análisis y el método empleado para comprobar la incidencia de las variables independientes. Se continúa con los resultados obtenidos del análisis empírico que sirve como antecedente a la sección de discusión sobre los hallazgos. Finalmente se exponen las conclusiones y las consideraciones finales.

\section{Definición de empresas zombis}

El término "zombi" ha sido empleado, en mayor medida, en el área de las finanzas, Kane (1989) lo utilizó por primera vez para describir a una institución bancaria en Estados Unidos afectada gravemente por la crisis de la década de 1980 y que se mantuvo operando gracias al apoyo directo de las instituciones gubernamentales (Tan et al., 2016). Posteriormente, el término fue usado para referirse a algunos tipos de préstamo bancario (financiamiento con pagos de interés sumamente bajos o con subvenciones) (Caballero et al., 2008). Denotando de esta forma que el calificativo de "zombi" está directamente relacionado con la mala asignación de recursos (Iwaisako et al., 2013).

Hoshi (2000) sostuvo que éstas prácticas pervertían la economía al evitar una "destruc- 
ción creativa", afectando a las economías y los mercados en Japón. Adicionalmente señaló que éste comportamiento seria transmitido de las instituciones a las empresas o imitado por éstas últimas con la finalidad de mantenerse operando (Caballero et al., 2008; Fukuda \& Nakamura, 2011). Las firmas zombis son definidas como aquellas empresas insolventes que se mantienen en los mercados por un flujo de financiamiento constante (Hoshi, 2006; Imai, 2016; Tan et al., 2016).

En otras palabras, son unidades económicas que tienen graves problemas para cumplir constantemente las obligaciones derivadas de la estructura de capital elegida por la administración. Su situación se ve agravada por una mala asignación de los recursos financieros obtenidos, lo cual genera un estancamiento o disminución en la producción y, por ende, de sus ingresos, repercutiendo en sus utilidades operativas (Caballero et al., 2008; Iwaisako et al., 2013; McGowan et al., 2016).

Otro mecanismo para asistir a éstas empresas, es mediante el apoyo directo de instituciones oficiales en forma de proyectos con valores nominales muy superiores a lo que realmente correspondería (Tan et al., 2016), sin embargo ésta vía es difícil de identificar. Independiente de la vía de apoyo éste tipo de firmas generan una saturación de mercado que interfiere en las operaciones de las empresas sanas que se ven obligadas a competir con ellas (McGowan et al., 2016).

En el caso de México, pese a no existir antecedentes sobre éste tipo de empresas en el país se tiene que, a partir de la liberación de la banca se instalaron bancos de capital extranjeros, lo que generó un aumento en la cartera de préstamos de hasta un $20 \%$ (López, 2015) sin tener control sobre los solicitantes de los mismos, lo que llevo a financiar a clientes con una capacidad de endeudamiento (Hazera, Hernandez, \& Quirvan, 2009; Marois, 2011).

De ésta forma, se concluye que las firmas zombis se caracterizan principalmente por un flujo de capital con un costo muy bajo, el cual puede ser en forma de tasas de interés preferenciales, concesiones ante pagos en mora, reducción de deudas, entre otras (Caballero et al., 2008), solicitados para evitar costos sociales (despido masivo de trabajadores) y económicos como un proceso de bancarrota (McGowan et al., 2016).

\section{Revisión empírica. Predictores de las empresas zombis}

Puesto que la conceptualización de empresa zombi enmarca a organizaciones que requieren y reciben apoyos de entidades externas, se estudian los elementos que llevan a una firma a tener el comportamiento descrito. Como punto de partida, la literatura enmarca características claras sobre el comportamiento de las empresas zombis, entre ellas se encuentra la falta de capacidad para poder competir adecuadamente en los mercados, especialmente en las industrias dinámicas (Caballero et al., 2008), así como la capacidad para generar flujos de efectivo en el corto plazo y no un rendimiento sostenible (Hoshi, 2006; Imai, 2016; Tan et al., 2016).

Para comprender la relación entre el rendimiento empresarial y las empresas zombis se emplea la visión basada en recursos, la cual plantea que los elementos con los que cuenta la firma, así como las habilidades para explotarlos, son los que colocan a las organizaciones en posiciones vanguardistas para obtener mejores resultados que la competencia, generando un efecto diferenciador entre ellas, denotando que el mercado donde opera está directamente involucrado con éste concepto (Ferreira, Marques, \& Azevedo, 2011; Gupta, Malhotra, Czinkota, \& Foroudi, 2016; Peña-Vinces, Casanova, Guillen, \& Urbano, 2017)

Partiendo de ésta visión, se puede ver el resultado empresarial desde dos perspectivas, la que se relaciona con la eficiencia en el manejo de actividades relacionadas con la administración y utilización de los recursos con los que se cuenta (costos, producción, inversión, entre otros) y, por otro lado, la cuota de mercado que maneja cada unidad comparada con los demás integrantes del sector, lo anterior permite tener un mejor entendimiento de la dinámica del mercado (Sauka, 2014; Stojcic, Hashi, \& Telhaj, 2013; Subramanian 
\& Reddy, 2012).

En síntesis, la visión basada en recursos argumenta que la capacidad de una organización para emplear sus recursos las lleva a obtener una determinada rentabilidad. Puntualizando en las capacidades empresariales, Shen y Chen (2017) explicaron que las empresas zombis no utilizan adecuadamente los activos productivos, inclusive, los subutilizan, perjudicando su estabilidad financiera, por lo cual la necesidad del auxilio por terceras instituciones crece mayormente.

La aportación anterior refuerza lo concluido por Hoshi (2006) quien encontró que, tanto el rendimiento operativo como el financiero, se relacionan fuertemente con la condición zombi. Entre los autores más recientes, se puede hacer referencia a Tan, Huang y Woo (2016) quien encuentra resultados en el mismo sentido, al igual que Iwaisako et al. (2013), Jiang, Li y Song (2017), McGowan et al. (2016) y Urionabarrenetxea et al. (2018).

Debido a los resultados empíricos obtenidos por los autores anteriores, para el presente artículo se espera que el rendimiento organizacional tenga un efecto negativo en las empresas zombis; además ofrece la oportunidad a las firmas de reinvertir a través de nuevos y mejores activos, una mayor fuerza laboral, mano de obra de mayor cualificación, entre otros elementos, lo que puede ser tomado como un resultado directo del nivel de competitividad organizacional (Akben-Selcuk, 2016; Kovacs, 2012; Man, Lau, \& Chan, 2002). Por lo anterior, se establece la siguiente hipótesis:

H1: El rendimiento empresarial afecta negativamente la probabilidad de que una organización sea zombi.

Otro elemento recurrente en la literatura especializada como un fuerte predictor de las empresas zombis es la dinámica del sector donde se encuentran estas organizaciones (Caballero et al., 2008; Fukuda \& Nakamura, 2011; McGowan et al., 2016). Puntualmente, una baja competencia en un sector favorece a que las empresas estancadas se mantengan en la industria, pues no requerirán tomar acciones distintas para competir; por lo tanto, tenderán a mantener posiciones pasivas en sus actividades. En otras palabras, los mercados con pocos niveles de competitividad son entornos muy favorables para la proliferación de empresas zombis (Fukuda \& Nakamura, 2011; McGowan et al., 2016).

Caballero et al. (2008) definió que los sectores con mayor actividad como las industrias tenían una menor presencia de empresas zombis, mientras que sectores como la construcción, el inmobiliario y la minería eran propicios para este tipo de empresas; en éste aspecto, Hoshi (2006) ofrece resultados similares. Puntualmente, Tan, Huang y Woo (2016) midió el dinamismo de la industria mediante las cuotas de mercado que mantenía cada organización; determinó que un mercado en donde existía una alta concentración era una industria poco competida, mientras que, un mercado competido es aquel en donde un gran número de empresas se disputan los márgenes de mercado.

En éste sentido, la presencia de firmas zombis en los mercados con mayor competencia y mayor productividad es baja, dado que altos niveles competitivos, obligan a mejorar las actividades, así como invertir en distintos rubros, tales como $\mathrm{I}+\mathrm{D}$, innovación, aumento en fuerza de ventas, inversión en activos, entre otros (Caballero et al., 2008; Fukuda \& Nakamura, 2011; McGowan et al., 2016); esto quiere decir que, las cuotas del mercado están divididas entre los competidores.

Por otro lado, un sector con baja competencia es un mercado concentrado, que propicia que las empresas estancadas se mantengan en el mercado, ya que no son obligada a realizar cambios en su estrategia empresarial y no se ven forzadas a plantear la posibilidad de abandonar el mercado (Gupta et al., 2016; Peña, Cepeda, \& Chin, 2012; Perez-Bustamante, Marques, Jalali, \& Ferreira, 2016).Por los antecedentes expuestos en este apartado, se espera que una alta concentración de mercado, que representa un mercado poco competido, afecte positivamente a la empresa zombi. A razón de lo anterior se define la siguiente hipótesis: 
H2: La concentración de mercado aumenta la probabilidad de que una empresa sea zombi.

Lo anterior, permite plantear el siguiente modelo de investigación (ver Figura 1) con la finalidad de realizar un acercamiento a los factores intrínsecos de las firmas que generan la condición zombi. En este caso, la selección de los elementos se realizó en base a la literatura empírica existente, autores como Caballero et al. (2008), Hoshi (2006), Imai (2016) y Tan et al. (2016) demostraron que son dos de las principales variables que explican la existencia de la empresa zombi, además, cada una de ellas contiene información sobre distintos elementos, es decir, el rendimiento esta estrechamente relacionado con la forma en que la organización realiza las actividades y que tan bien lo hace (Gupta et al., 2016; Peña-Vinces et al., 2017); mientras que la cuota de mercado captura información sobre las características de la industria donde operan (Li, Nie, Zhao, \& Li, 2017; Martin, Upham, \& Budd, 2015; Wu, Gao, \& Gu, 2015).

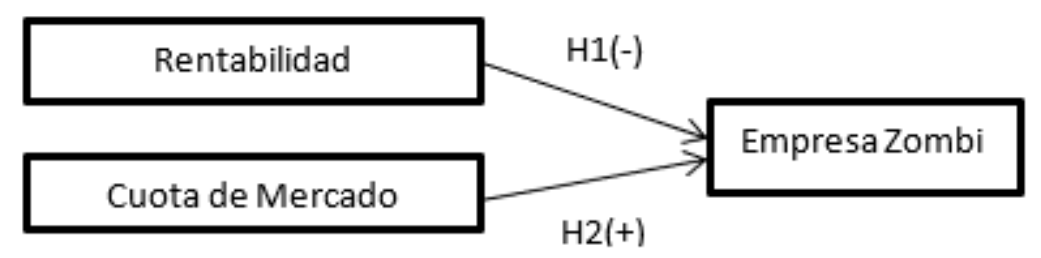

Figura 1. Modelo de competitividad de las empresas zombis. Fuente: elaboración propia.

\section{Diseño Metodológico}

\subsection{Unidad de análisis}

Para poder cumplir con los objetivos trazados en esta investigación se seleccionaron a empresas que operan en los mercados de capitales, particularmente se circunscribe a firmas que cotizan en la Bolsa Mexicana de Valores (BMV). La población de empresas inscritas en la BMV es de 147 empresas distribuidas en nueve sectores de distintas actividades; cabe aclarar que no fueron tomadas en cuenta dentro de la muestra aquellas organizaciones que ofrecen servicios financieros y de seguros como su actividad principal. La razón por la cual se excluyeron estas empresas es debido a que reportan información que difiere, en forma, de las firmas que desempeñan otra actividad, lo cual provoca que estas empresas no puedan ser comparada.

Una vez realizada la extracción las empresas del sector financiero, se conformó una muestra de 102 empresas de ocho sectores distintos. Estas organizaciones están obligadas a hacer pública su información financiera, la cual puede ser consultada en la página web de la Bolsa Mexicana de Valores ${ }^{1}$, dichos reportes contienen los estados financieros básicos, así como el número de empleados e información histórica de la empresa; de este reporte es de donde se extrajo la información pertinente para la construcción de las variables de estudio que se explicarán a continuación. La información financiera utilizada para la conformación de la base de datos corresponde a las actividades del año 2015. Por lo anterior, la muestra estuvo conformada de la siguiente forma:

\footnotetext{
${ }^{1}$ La información de cada empresa puede ser consultada en la siguiente dirección web: https://www.bmv.com.mx/es/emisoras/informacion-de-emisoras
} 
Tabla 1. Número de empresas por sector

\begin{tabular}{|l|c|}
\hline \multicolumn{1}{|c|}{ Clasificación por Sector } & Número de empresas \\
\hline ENERGIA & 1 \\
\hline INDUSTRIAL & 31 \\
\hline MATERIALES, MATERIAS PRIMAS & 22 \\
\hline PRODUCTOS DE CONSUMO FRECUENTE & 15 \\
\hline SALUD & 5 \\
\hline SERVICIOS DE TELECOMUNICACIONES & 10 \\
\hline SERVICIOS Y BIENES DE CONSUMO NO BASICO & 17 \\
\hline TECNOLOGIAS DE LA COMUNICACIÓN. & 1 \\
\hline \multicolumn{2}{|c|}{ TOTAL } \\
\hline
\end{tabular}

Fuente: Elaboración propia.

\subsection{Método}

\subsubsection{Definición de Variable Dependiente}

Para identificar a las organizaciones como empresas zombis se emplea la medida zombi propuesta por Caballero, Hoshi y Kashyap (2008) conocido como método CHK, que evalúa si una organización recibe un trato preferencial en su financiamiento, es decir, si es apoyada por agentes externos para operar. Lo anterior se determina mediante el cálculo del costo del financiamiento mínimo $\left(\mathrm{Ri}^{*}\right)$ para ello se emplea la tasa libre de riesgo, la deuda a corto plazo (CP) y deuda a largo plazo (LP) (Fukuda \& Nakamura, 2011; Hoshi, 2006; Imai, 2016; Iwaisako et al., 2013; Tan et al., 2016).

$$
R_{i}{ }^{*}=r s_{t-1} C P_{i, t-1}+\left(\frac{1}{5} \varepsilon r l_{t-j}\right) \mathrm{LP}_{i, t-1}
$$

En donde:

$\mathrm{CP}=$ pasivos a corto plazo menos cuentas por pagar, impuestos a pagar y otros elementos pagaderos para medir la deuda bancaria a corto plazo.

$\mathrm{LP}=$ Pasivos a largo plazo.

$\mathrm{rs}=$ Promedio del porcentaje de interés a corto plazo.

$\mathrm{rl}=$ Promedio del porcentaje de interés a largo plazo.

Finalmente, se realiza una diferencia entre el costo de financiamiento real $\left(R_{i}\right)$ y el costo mínimo calculado $\left(\mathrm{R}^{*}\right)$; dicha operación se normaliza al dividirlo entre el monto total de deuda del año inmediato anterior, a continuación su expresión matemática:

$$
\operatorname{gap}_{\mathrm{i}, \mathrm{t}}=\mathrm{R}_{\mathrm{i}}-\mathrm{R}_{\mathrm{it}-1}^{*}
$$

En donde:

$\mathrm{R}=$ pago real de intereses de la empresa.

$R^{*}=$ pagodeinter smnimoesperado.

$\mathrm{B}_{i t-1}=$ préstamos del periodo anterior.

Puesto que el modelo logit tradicional implica que la variable resultado es una categoría dicotómica, es decir, si la observación cuenta o no con una condición (Cameron \& Trivedi, 2010); para ésta investigación, la variable dependiente toma los siguientes valores:

$$
y= \begin{cases}1 & \text { si gap }<0 \\ 0 & \text { si } \text { gap } \geq 0\end{cases}
$$

En donde 1 corresponde a la empresa categorizada como zombi, mientras que 0 es para las empresas normales. 


\subsubsection{Definición de Variables independientes}

De acuerdo con la literatura especializada en empresas zombis, las características que son asociadas normalmente a esta condición son la competitividad de los mercados y el rendimiento de la organización (Caballero et al., 2008; Hoshi, 2006; Iwaisako et al., 2013; Jiang et al., 2017; McGowan et al., 2016; Urionabarrenetxea et al., 2018). Tomando como referencia lo anterior, se procede a medir el nivel de concentración de mercado como variable que representa el nivel de competitividad.

La decisión se utilizar el grado de concentración del mercado se basa en que dicha medida, que se refiere a la parte de las ventas realizadas por una empresa dentro de una industria; de forma práctica, representara el nivel en el que el mercado se concentra en una determinada organización (Schmalensee, 1985).

La medida tradicional para la medición del nivel de concentración de un mercado es el índice HH (Gallagher, Ignatieva, \& McCulloch, 2014; Gallagher et al., 2014; Go, Kamerschen, \& Delrome, 1999; Khan, Ahmad, \& Chan, 2018; Khan, Ahmad, \& Gee, 2016; Schmalensee, 1989); sin embargo, el resultado se obtiene a nivel industria y se desea trabajar con información a nivel empresa. La cuota relativa de mercado, además de ser una observación a nivel empresa, da información sobre el papel que desempeña cada organización en una determinada industria, es decir, además del grado de concentración, nos dice en qué posición se encuentra la empresa, dentro del mercado (Kimenyi, Lee, \& Shughart, 1990; Li et al., 2017; Martin, 1983, 1984; Shepherd, 1972; Wu et al., 2015). La fórmula para calcular la cuota relativa de mercado se expresa a continuación.

$$
\text { Cuota relativa de mercado }=\frac{X_{i j}}{X_{j}}
$$

En donde:

$X_{i j}=$ Ventas de la empresa i en la industria $\mathrm{j}$.

$X_{j}=$ Ventas de la industria $\mathrm{j}$.

A manera de resumen, se espera que un valor mayor representa una alta concentración del mercado y una mayor participación en el mismo; por otro lado, un resultado menor indica un mercado poco concentrado o, una baja participación en la industria por la empresa.

Por otro lado, la rentabilidad financiera de cada empresa se midió mediante la razón rendimiento sobre activos (ROA), la cual representa la capacidad productiva de los bienes de la firma. La razón de emplear este indicador es, en primera instancia, es uno de los principales variables utilizadas para predecir la bancarrota empresarial (CamachoMiñano, Segovia-Vargas, \& Pascual-Ezama, 2015); por otro lado, autores como Goto y Wilbur (2018) y, Iwaisako et al. (2013) encontraron empíricamente una relación estadística significativa y negativa con las empresas zombis. Además, el cálculo del ROA no utiliza información financiera empleada en el método CHK y se evitó el problema de multicolinealidad entre las variables dependientes.

\subsubsection{Técnica de análisis}

Una vez definidas las métricas sobre las variables de estudio, para la conformación de la base de datos, se revisó el balance general y el estado de resultados dictaminados, mismos que son públicos y se incluyen en el informe anual de cada una de las 102 empresas inscritas en la BMV objeto de este estudio.

Para medir la contribución de la concentración del mercado y la rentabilidad en la probabilidad de que la empresa sea zombi en México se realizó una regresión logística binaria. Esto debido a que es una técnica ampliamente usada en la literatura especializada sobre empresas zombis, Caballero, Hoshi y Kashyap (2008), Fukuda y Nakamura (2011), 
Goto y Wilbur (2018) y, Hoshi (2006) emplearon ésta técnica para comprobar los factores que inciden en las empresas zombis.

Lo anterior se debe, a que el modelo logit requiere de una distribución de Bernoulli (una variable dicotómica) para probar variables regresoras que modifiquen la probabilidad de encontrar o no determinada condición (Cameron \& Trivedi, 2010); el presente estudio adopta esta técnica puesto que se adaptan de forma adecuada a la diferenciación de las empresas zombis y las organizaciones que no lo son. Por lo anterior, se plantea el siguiente modelo econométrico:

$$
\operatorname{Pr}\left(\text { Zombi }_{i t} 1 \mid \text { ROA,CuotaMerc }\right)=\frac{1}{1+e^{\left(-\beta_{0}-\beta_{1} \chi_{1} \beta_{2} \chi_{2}\right)}}
$$

En donde:

$\operatorname{Pr}\left(Z o m b i_{i t} 1 \mid R O A\right.$, CuotaMerc) es la probabilidad de que la variable dependiente que corresponde a la medida zombi de cada empresa i y para cada año t tome el valor 1 en presencia de la covariable de ROA y CuotaMerc.

$\beta_{0}$ es la constante del modelo.

$\beta_{1}$ es el coeficiente de la covariable de la Cuota Mercado (CuotaMerc).

$\beta_{2}$ es el coeficiente de la covariable de la rentabilidad empresarial (ROA).

$\varepsilon$ es el posible error de predicción para cada covariable $\chi$

$e$ representa la constante de Euler $(2,718)$.

\section{Resultados}

De las 102 empresas objeto de estudio fueron categorizadas como empresa zombi, mediante el método CHK, 18 organizaciones (ver Figura 2) distribuidas en los sectores Energía, Industrial, Materiales y materias primas, Servicios de telecomunicación y Bienes de consumo no básico, tal como se aprecia en la tabla 2.

Tabla 2. Número de empresas por sector

\begin{tabular}{|l|c|c|}
\hline \multicolumn{1}{|c|}{ Clasificación por Sector } & Número de empresas & Empresas Zombis \\
\hline 1 ENERGIA & 1 & 1 \\
\hline 2 INDUSTRIAL & 31 & 6 \\
\hline 3 MATERIALES, MATERIAS PRIMAS & 22 & 7 \\
\hline 4 PRODUCTOS DE CONSUMO FRECUENTE & 15 & 0 \\
\hline 5 SALUD & 4 & 0 \\
\hline 6 SERVICIOS DE TELECOMUNICACIONES & 9 & 1 \\
\hline 8 SERVICIOS Y BIENES DE CONSUMO NO BASICO & 17 & 3 \\
\hline 9 TECNOLOGIAS DE LA COMUNICACIÓN. & 1 & 0 \\
\hline TOTAL & $\mathbf{1 0 2}$ & $\mathbf{1 8}$ \\
\hline
\end{tabular}

Nota: el sector 7 corresponde a las empresas financieras y aseguradoras.

Así mismo, se puede identificar que los sectores asociados con actividades primarias y secundarias (extracción y transformación, respectivamente) tienen mayor número de empresas zombis, lo cual es indicativo sobre el tipo de actividad que desarrolla estas organizaciones. 


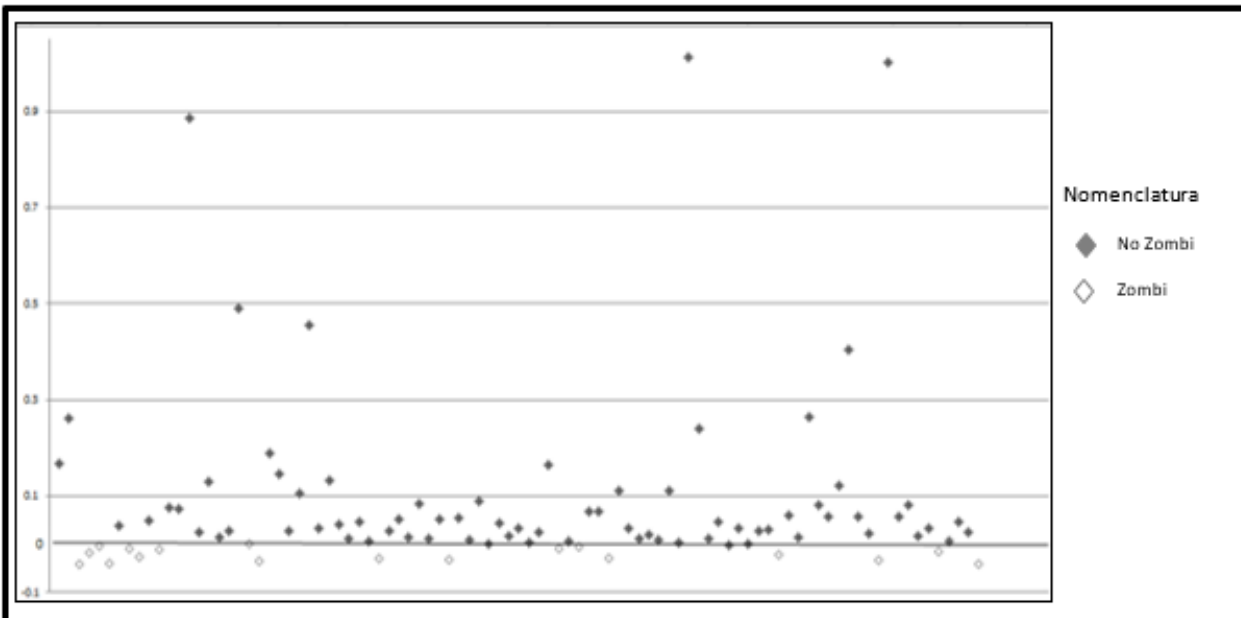

Figura 2. Clasificación de empresas zombis.

Nota: Clasificación elaborada a partir del cálculo de la medida CHK la cual, se encuentra representada en el eje.

Para profundizar más sobre el contexto de las organizaciones, tomando como base el estudio de McGowan et al. (2016), se realiza un análisis comparativo descriptivo sobre alguna de las características de las empresas zombis y no zombis de cada sector, particularmente en la cantidad de ventas, el número de empleados y la edad (ver figura 3, figura 4 y figura 5$)$.

Se encontró que las empresas zombis son las que tienen una mayor cantidad de ventas, indicativo de que pocas empresas ejercen un mayor dominio sobre el mercado. Por otro lado, no se aprecia una tendencia clara sobre la cantidad de empleados promedios por sector entre empresas zombis y no zombis. Sin embargo, se aprecia que en el sector de servicio de telecomunicaciones (sector 6) se aglutina, tanto un mayor promedio de ventas como un gran número de empleados, indicativo que pocas empresas son las que aglutinan la mayor cantidad de operaciones y que la competencia son actores limitados, propio de un oligopolio.

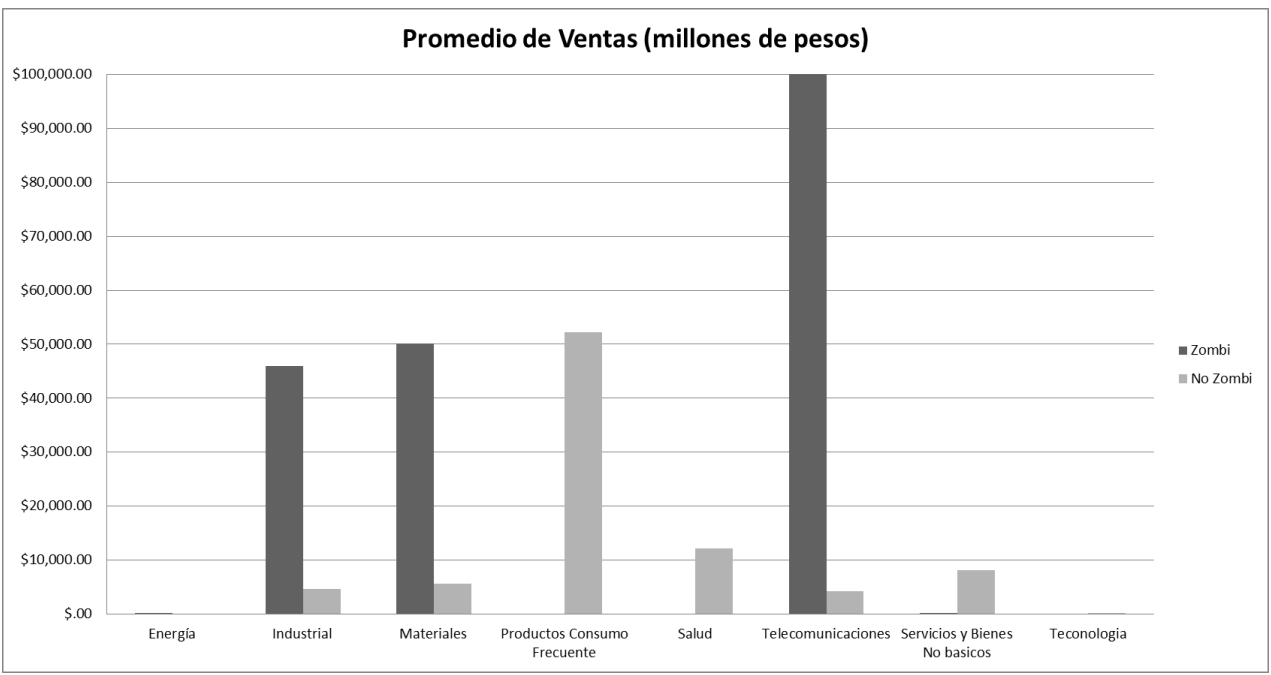

Figura 3. Promedio de Ventas de empresas zombis y no zombis.

Nota: Para elaborar la gráfica se promedió el volumen de venas en miles de pesos por cada sector. 


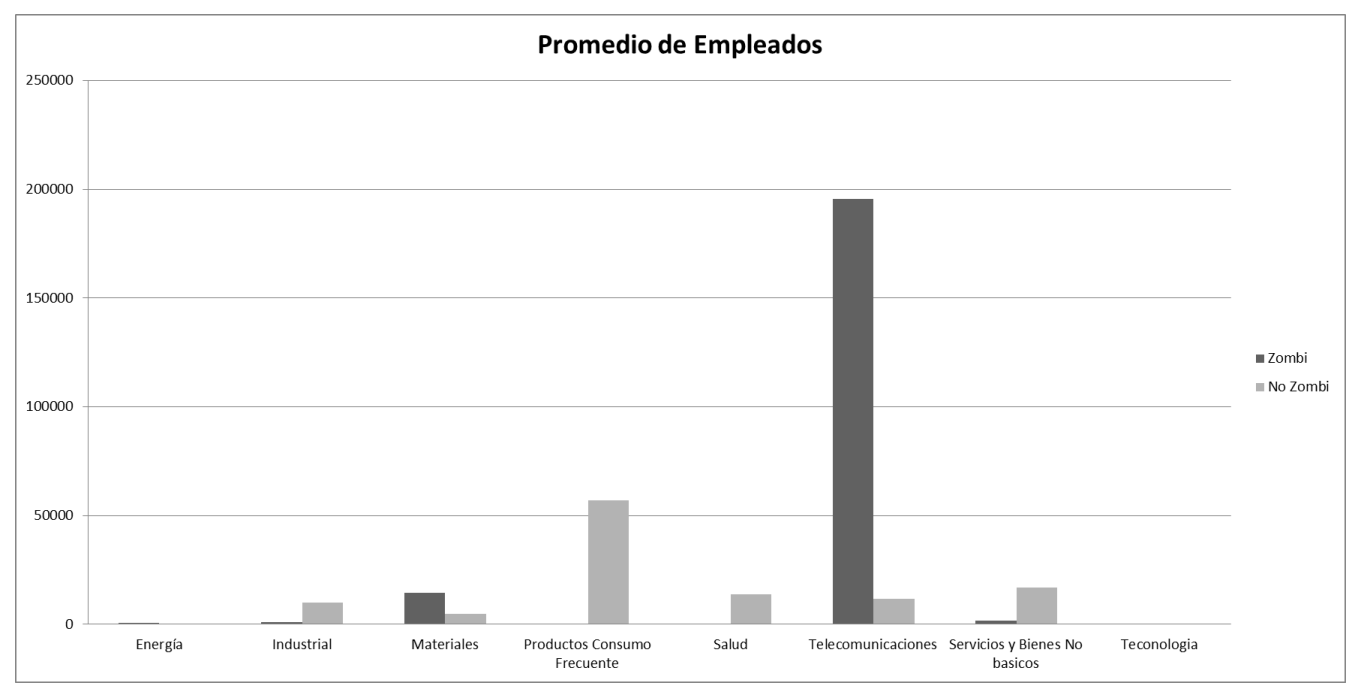

Figura 4. Promedio de empleados de empresas zombis y no zombis.

Nota: Para elaborar la gráfica se promedió el volumen de venas en miles de pesos por cada sector.

En su estudio sobre economías inscritas en la OCDE, McGowan et al. (2016) encontró que las empresas zombis tienden a tener un mayor periodo de vida que las otras, resultado que concuerda con los hallazgos en la muestra de este estudio, ya que las empresas con características zombis en México tienen, en promedio, un mayor periodo de vida (ver Figura 5).

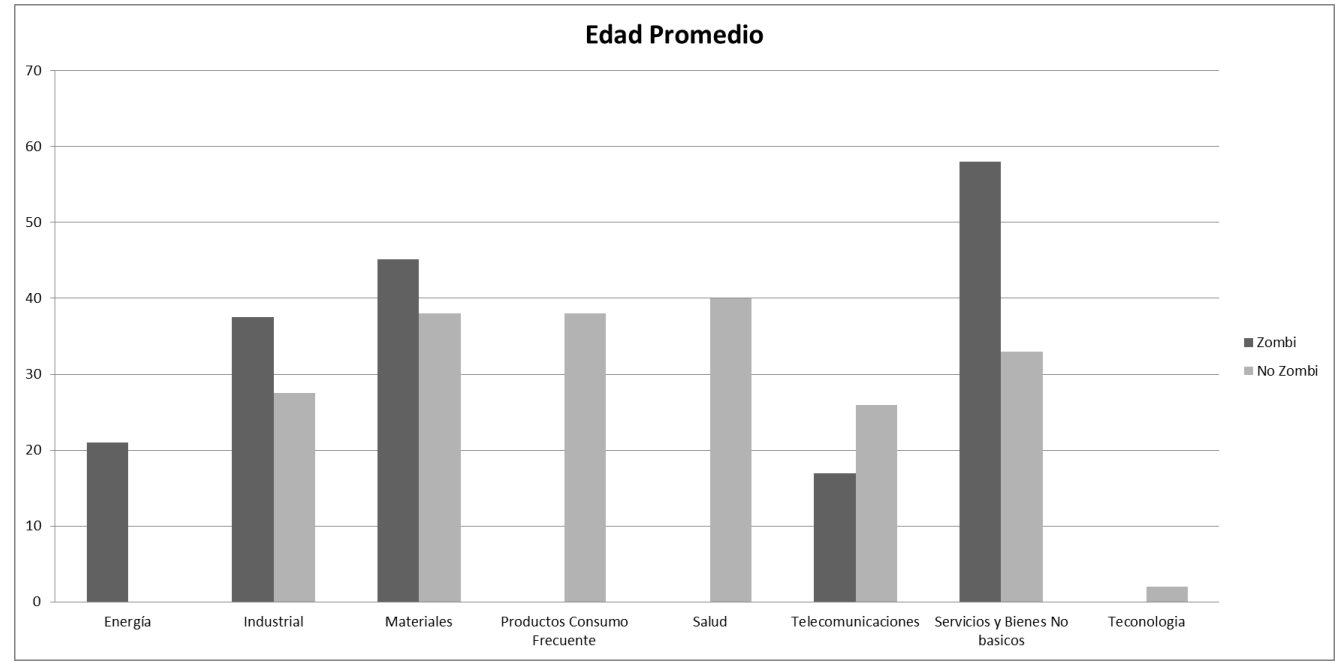

Figura 5. Edad promedio de empresas zombis y no zombis.

Nota: Para elaborar la gráfica se promedió el tiempo de vida de empresas por cada sector.

De lo anterior se concluye que las empresas zombis, en los sectores donde tienen presencia, son las que tienen un volumen de operaciones mayor a la competencia, lo que sugiere que éstos mercados pueden estar altamente concentrados en pocas empresas, es decir, están presentes en mercados con características oligopólicas lo que restringiría el dinamismo del sector y de las firmas que lo conforman.

Previo al análisis multivariante, la tabla 4 muestra el resumen de la estadística descriptiva para cada variable independiente en donde se aprecia una amplia disparidad entre las empresas que componen la muestra; en cuanto a la Cuota de Mercado se muestra aprecia 
existen empresas que se encuentran en industrias monopólicas, mientras que otras se encuentran en mercados con cuotas más divididas. En cuanto al ROA, existe una disparidad entre el rendimiento de las distintas empresas, asociándolo con la variable dicotómica de empresa zombi el análisis podrá ofrecer diferencias entre los tipos de empresas.

Tabla 3. Estadística descriptiva de las variables independientes

\begin{tabular}{|c|c|c|}
\hline & ROA & Cuota de Mercado \\
\hline Media & 0.78004 & 0.1061 \\
\hline Varianza & 0.442 & 0.68 \\
\hline Desv. Estándar & 0.664 & 0.261 \\
\hline Mínimo & 0.0072 & 0.000017 \\
\hline Máximo & 3.803 & 1.000 \\
\hline
\end{tabular}

Fuente: elaborado a partir de los datos obtenidos de los reportes anuales públicos de cada empresa que cotiza en la BMV

Para la predicción de catalogar una empresa zombi o no zombi, se utilizó una regresión logística categorizando la variable dependiente en empresas zombis (18 empresas) y empresas no zombis (84 empresas). El modelo permite hacer una estimación correcta total del $85.3 \%\left(\chi^{2}=15.103, \mathrm{p}<.01\right)$, siendo los predictores de la ecuación el rendimiento financiero "ROA" (Wald $=3.627, \mathrm{p}=0.057$ ) y cuota de mercado "CuotaMerc" (Wald $=9.997, \mathrm{p}=0.002$ ).

En cuanto a los valores obtenidos, el modelo logit (Pr Zombi CuotaMerc,ROAit $=1 \mid$ CuotaMerc,ROA) alcanzan una probabilidad predicha de 0,745 (como es mayor de 0,50 ) implica que las empresas con una mayor cuota relativa de mercado y un ROA menor tienen una mayor posibilidad de ser clasificadas como empresas Zombi (Ver Tabla 4).

Tabla 4. Resumen del modelo.

\begin{tabular}{|l|c|c|c|}
\hline & Coeficiente & Sig & Exp B \\
\hline Constante & -1.251 & .000 & .286 \\
\hline CuotaM & 3.458 & .002 & 31.759 \\
\hline ROA & -1.137 & .057 & .321 \\
\hline Chi-cuadrado & 15.103 & & \\
\cline { 1 - 2 } Predicciones Correctas & $85.3 \%$ & \\
\cline { 1 - 2 } Número de Observaciones & 102 & \\
\cline { 1 - 1 } R2L $=.177 ;$ R2CS $=.137 ; \mathrm{R} 2 \mathrm{~N}=.226 ; \mathrm{x} 2=15.061 ; \mathrm{p}=.001$ \\
\hline
\end{tabular}

Nota: $R^{2} \mathrm{~L}=\mathrm{R}$ cuadrado logística; $R^{2} \mathrm{CS}=\mathrm{R}$ cuadrado de Cox Snell; $R^{2} \mathrm{~N}=\mathrm{R}$ cuadrado de Nagelkerke.

La participación del ROA en la probabilidad de clasificación como empresa zombi debería de interpretarse con cautela, puesto que dicha variable supera levemente el valor p de .05 de significatividad, lo cual es evidencia que soporta parcialmente H1. Siguiendo con el análisis individual, la variable ROA presenta un signo negativo en su coeficiente, lo que indica que un aumento en ella se traducirá en una disminución en la probabilidad de que la empresa sea zombi. En este sentido, podemos interpretar que las empresas al tener un mayor rendimiento sobre los activos tienen menor probabilidad de ser zombi; en cuanto a la cuota de mercado la relación es contraria, es decir, que, al aumentar la cuota de mercado, la probabilidad de ser zombi aumenta, dicho resultado estadístico es evidencia que no permite rechazar $\mathrm{H} 2$.

\section{Discusión}

En primera instancia, el presente trabajo corrobora la existencia de empresas zombis en México, además indaga sobre el origen de ellas. Si bien es cierto las primeras investigaciones diagnosticaron a estas empresas a causa de una crisis financiera, la evidencia sugiere 
que estas organizaciones no son originadas por este fenómeno económico, sino que, tal y como menciona Urionabarrenetxea et al. (2018), su origen está en cuestiones estructurales y organizativas.

Realizando un contraste con las referencias empíricas sobre empresas zombis se obtuvo evidencia, que concuerda con los resultados mostrados por Caballero et al. (2008), Hoshi (2006) y Tan et al. (2016), es decir, la rentabilidad tiene un efecto negativo con las empresas zombis. Cabe señalar que los estudios anteriores se circunscriben a economías asiáticas, esto quiere decir que las empresas zombis de esa región son similares a las latinoamericanas, lo que va acorde a lo descrito por Shen y Chen (2017) y Urionabarrenetxea et al. (2018) quienes argumentaron que estas organizaciones tienen su origen en problemas operativos o estructurales, y no precisamente en eventos macroeconómicos o financieros.

Lo anterior se refuerza con lo planteado por Andrews, Criscuolo y Gal (2016); Caballero, Hoshi y Kashyap (2008); Hoshi (2006) McGowan, Andrewsn y Millot (2016), quienes sostienen que los mercados con características oligopólicas o con menor competencia son más propicios para la existencia de empresas zombis, en este caso, las altas cuotas de mercado en una industria implican una alta concentración de ventas en pocas firmas, lo que disminuye la actividad de las demás y limita el desarrollo, la innovación, la entrada de nuevos negocios, entre otros. Por ello, en futuras investigaciones deberán incluirse medidas de concentración de mercado para poder estudiar este efecto de forma directa con las empresas zombis.

Además, cabe mencionar que autores como Andrews, Criscuolo, \& Gal (2016) y McGowan, Andrews y Millot (2016) encontraron que las empresas zombis son organizaciones que suelen ser grandes entidades económicas, con un alto número de empleados y que tienen más antigüedad, característica que comparte con las empresas zombis encontradas en este estudio. Las implicaciones de los hallazgos se exponen a continuación.

Retomando la relación negativa encontrada en este estudio entre el rendimiento y las empresas zombis, indica que, si una empresa no desarrolla sus actividades de forma adecuada, eventualmente, será una empresa zombi. Sin embargo, el punto no es debatir sobre la adecuada operación de determinada empresa, sino por qué pese a tener mal rendimiento estás organizaciones siguen en el mercado.

Parte de la explicación se encuentra en la industria donde opera, por lo cual, el diagnóstico y la descripción de la unidad de análisis es fundamental para entender, además del uso de la concentración del mercado como variable explicativa nos da información adicional sobre ello.

Recordando que los sectores Materiales, Bienes de consumo no básico y Telecomunicaciones son en donde las empresas zombis cuentan con una mayor participación, lo cual significa que su existencia es necesaria para mantener la dinámica económica y comercial, es decir, son organizaciones que se encargan de desarrollar infraestructura, ofrecer servicios o productos necesarios para consumidores intermedios o finales.

Como segundo punto, autores como Caballero, et al., (2008), Hoshi (2006) e Imai (2016) encontraron que estas firmas afectan negativamente, tanto la productividad del sector como a las empresas "sanas"", aclarando que este trabajo no indaga sobe los efectos que producen estas empresas en los distintos mercados, sin embargo la evidencia sugiere que las firmas zombis no solo compiten contra las demás organizaciones sino que acaparan al menos dos factores de producción, capital (al recibir financiamiento a un costo menor) y trabajo (al concentrar mayor número de trabajadores), lo que puede ser considerado como efecto negativo.

Debido a lo anterior, es importante proponer que institucionalmente se implementen o mejoren las políticas que fomenten la competitividad de las organizaciones, mejorando las habilidades en prospectiva, planeación, ejecución y monitoreo por parte de la dirección y, no solo se les dote de recursos como medida de respaldo para mantener su existencia. Normalmente se pensaría que las empresas zombis son organizaciones que deberían salir 
del mercado, sin embargo, considerando sus características de amplia infraestructura o capital y el número de empleados que las componen, son empresas que resultan necesarias para atender otras industrias pues las empresas zombis les proporcionan insumos y facilitan la actividad comercial del tejido empresarial.

Para que este tipo de organizaciones se recuperen de su condición es necesario prestar especial atención a la falta de competitividad en los sectores producticos de las economías en América Latina para atenderlos y que se transformen en un motor de arrastre que coadyube al desarrollo de la región.

\section{Conclusiones y consideraciones finales}

Esta investigación propuso identificar a empresas mexicanas que cotizan en la BMV con problemas de insolvencia mediante la medida zombi CHK, con el cual se determinó que 18 de las 102 firmas que comprendieron el estudio cumplían con los requisitos determinados para su categorización como zombi. En este sentido, se encontró que existe un mayor número de empresas zombi en los sectores asociados con la industria, sin embargo, es en el sector servicios (especialmente en el sector telecomunicaciones) donde se aprecia un mayor peso en el mercado de las empresas con condición zombi al acaparar un mayor volumen de ventas y de trabajadores (indicativo de una alta concentración en el mercado) del sector.

En resumen, los resultados muestran que una empresa que cuenta con un mayor rendimiento financiero tendrán una menor probabilidad de encontrarse en la condición definida como firma zombi, de lo cual se concluye que las habilidades de las organizaciones para producir de mejor forma, fuerza de ventas sobresaliente, contar con mejores procesos y mano de obra calificada, entre otros, son fundamentales para el correcto funcionamiento empresarial (Chuang \& Huang, 2015; Ferreira et al., 2011; Peña et al., 2012; Solleiro \& Castañón, 2005, 2005).

Por otro lado, al analizar la influencia que ejerce la cuota de mercado sobre la aparición de empresas zombis se encontró una relación positiva entre ellas, lo cual quiere decir que, a medida que una empresa acapara el mercado tiene mayor probabilidad de convertirse en empresa zombi, la idea se clarifica al tomar en cuenta que, una empresa que tiene un amplio dominio del mercado representa una industria oligopólica, en este sentido, una industria oligopólica tiene mayor probabilidad de mantener empresas zombi.

Considerando lo anterior, el presente artículo es de las primeras contribuciones sobre el estudio de empresas zombis en América Latina, con motivo de comprender a estas organizaciones particulares identificando que son aspectos internos y externos los que contribuyen a la situación zombi.

Por último, con la finalidad de mejorar la comprensión de estas empresas particulares, especialmente en América Latina, se abren nuevas líneas de investigación. Tal es el caso, de profundizar en otros factores internos que puedan explicar en mayor medida a la empresa zombis como la gestión y dirección estratégica de las mismas. Sin olvidar que deben analizarse adicionalmente aquellos elementos o condiciones del mercado que puedan repercutir en la generación de una condición zombi. 
REMEF (The Mexican Journal of Economics and Finance)

\section{Referencias}

Akben-Selcuk, E. (2016). Factors Affecting Firm Competitiveness: Evidence from an Emerging Market. International Journal of Financial Studies, 4(2), 9. https://doi.org/10.3390/ijfs4020009

Amato, M., \& Fantacci, L. (2016). Failures on the market and market failures: a complementary currency for bankruptcy procedures. Cambridge Journal of Economics, 40(5), 1377-1395. https://doi.org/ $10.1093 / \mathrm{cje} /$ bew029

Andrews, D., Criscuolo, C., \& Gal, P. (2016). The Best versus the Rest: The Global Productivity Slowdown, Divergence across Firms and the Role of Public Policy. Paris: OECD Publishing.

Caballero, R. J., Hoshi, T., \& Kashyap, A. K. (2008). Zombie Lending and Depressed Restructuring in Japan. American Economic Review, 98(5), 1943-1977. https://doi.org/10.1257/aer.98.5.1943

Camacho, M., Segovia, M., \& Pascual, D. (2015). Which Characteristics Predict the Survival of Insolvent Firms? An SME Reorganization Prediction Model. Journal of Small Business Management, 53(2), 340-354. https://doi.org/10.1111/jsbm.12076

Camacho-Miñano, M., Segovia-Vargas, M.-J., \& Pascual-Ezama, D. (2015). Which Characteristics Predict the Survival of Insolvent Firms? An SME Reorganization Prediction Model. Journal of Small Business Management, 53(2), 340-354. https://doi.org/10.1111/jsbm.12076

Cameron, A., \& Trivedi, P. (2010). Microeconometrics using stata (Revised). Estados Unidos: Stata Press.

Chuang, S.-P., \& Huang, S.-J. (2015). Effects of Business Greening and Green IT Capital on Business Competitiveness. Journal of Business Ethics, 128(1), 221-231. https://doi.org/10.1007/ s10551-014-2094-y

Ferreira, J. J., Marques, C. S., \& Azevedo, C. (2011). Competitiveness, resources, and capabilities: empirical evidence from retail banking. Service Business, 5(4), 313-337. https://doi.org/10.1007/ s11628-011-0117-7

Fukuda, S., \& Nakamura, J. (2011). Why Did "Zombie" Firms Recover in Japan? World Economy, 34(7), 1124-1137. https://doi.org/10.1111/j.1467-9701.2011.01368.x

Gallagher, D. R., Ignatieva, K., \& McCulloch, J. (2014). Industry concentration, excess returns and innovation in Australia. Accounting \& Finance, 55(2), 443-466. https://doi.org/10.1111/acfi. 12074

Go, G., Kamerschen, D., \& Delrome, C. (1999). Market structure and price-cost margins in Philippine manufacturing industries. Applied Economics, 31(7), 857-864. https://doi.org/10.1080/ 000368499323814

Goto, Y., \& Wilbur, S. (2018). Unfinished business: Zombie firms among SME in Japan's lost decades. Japan and the World Economy. https://doi.org/10.1016/j.japwor.2018.09.007

Gupta, S., Malhotra, N. K., Czinkota, M., \& Foroudi, P. (2016). Marketing innovation: A consequence of competitiveness. Journal of Business Research, 69(12), 5671-5681. https://doi.org/10.1016/ j.jbusres.2016.02.042

Hazera, A., Hernandez, S. M., \& Quirvan, C. (2009). Toward a conceptual framework of the harmonization of bank financial reporting standards in transition economies: The case of Mexico. Advances in Accounting, 25(2), 237-254. https://doi.org/10.1016/j.adiac.2009.09.002

Hoshi, T. (2000). Why is the Japanese economy unable to get out of a liquidity trap?, 233-266.

Hoshi, T. (2006). Economics of the living dead. Japanese Economic Review, 57(1), 30-49. https://doi. org $/ 10.1111 / j .1468-5876.2006 .00354 . x$

Imai, K. (2016). A panel study of zombie SMEs in Japan: Identification, borrowing and investment behavior. Journal of the Japanese and International Economies, 39, 91-107. https://doi.org/10. 1016/j.jjie.2015.12.001

Ito, T., \& Sasaki, Y. N. (2002). Impacts of the Basle capital standard on Japanese banks' behaviors. Journal of the Japanese and International Economies, 16(3), 372-397. https://doi.org/10.1006/ jjie.2002.0509

Iwaisako, T., Fukuoka, C., \& Kanou, T. (2013). Debt Restructuring of Japanese Corporations: Efficiency of Factor Allocations and the Debt-Labor Complementarity. Hitotsubashi Journal of Economics, 54(1), 119-135.

Jiang, X., Li, S., \& Song, X. (2017). The mystery of zombie enterprises - "stiff but deathless". China Journal of Accounting Research, 10(4), 341-357. https://doi.org/10.1016/j.cjar.2017.08.001

Kane, E. (1989). The S \& L Insurance Mess: How Did It Happen? (The Urban Insitute). Washington, DC.

Khan, H. H., Ahmad, R. B., \& Chan, S. G. (2018). Market structure, bank conduct and bank performance: Evidence from ASEAN. Journal of Policy Modeling, 40(5), 934-958. https://doi.org/10.1016/j . jpolmod.2018.02.001

Khan, H. H., Ahmad, R. B., \& Gee, C. S. (2016). Market Structure, Financial Dependence and Industrial Growth: Evidence from the Banking Industry in Emerging Asian Economies. PLOS ONE, 11(8), e0160452. https://doi.org/10.1371/journal.pone.0160452

Kimenyi, M. S., Lee, J., \& Shughart, W. F. (1990). Price-Cost Margins and Industry Structure in Developing Countries: The Case of Korea*. Bulletin of Economic Research, 42(3), 197-210. https: //doi.org/10.1111/j.1467-8586.1990.tb00670.x 
Kovacs, Z. (2012). The Key to Competitiveness: Forecast. Acta Oeconomica, 62(4), 505-518. https: //doi.org/10.1556/ADecon.62.2012.4.5

Li, Y., Nie, D., Zhao, X., \& Li, Y. (2017). Market structure and performance: An empirical study of the Chinese solar cell industry. Renewable and Sustainable Energy Reviews, 70, 78-82. https: //doi.org/10.1016/j.rser.2016.11.064

López, J. (2015). Trade and financial liberalization revisited: Mexico's experience. Journal of Post Keynesian Economics, 38(3), 376-398. https://doi.org/10.1080/01603477.2015.1087809

Man, T. W. Y., Lau, T., \& Chan, K. F. (2002). The competitiveness of small and medium enterprises: A conceptualization with focus on entrepreneurial competencies. Journal of Business Venturing, 17(2), 123-142. https://doi.org/10.1016/S0883-9026(00)00058-6

Marois, T. (2011). Emerging market bank rescues in an era of finance-led neoliberalism: A comparison of Mexico and Turkey. Review of International Political Economy, 18(2), 168-196. https://doi. org/10.1080/09692290903475474

Martin. (1983). Market, Firm, and Economic Performance (1a ed.). Estados Unidos: New York University Graduate School of Business Administration, Salomon Brothers Center for the Study of Financial Institutions.

Martin. (1984). Testing the interaction between concentration and barriers to entry. Review of Industrial Organization, 1(2), 114-126. https://doi.org/10.1007/BF02354347

Martin, C. J., Upham, P., \& Budd, L. (2015). Commercial orientation in grassroots social innovation Insights from the sharing economy. Ecological Economics, 118, 240-251. https ://doi.org/10.1016/ j.ecolecon.2015.08.001

McGowan, A., Andrews, D., \& Millot, V. (2016). The Walking Dead? Zombie firms and Productivity Performance in OECD countries. OECD Economics Departament Working Papers.

Peña, J., Cepeda, G., \& Chin, W. (2012). Effect of ITC on the international competitiveness of firms. Management Decision, 50(6), 1045-1061. https://doi.org/10.1108/00251741211238328

Peña-Vinces, J. C., Casanova, L., Guillen, J., \& Urbano, D. (2017). International Competitiveness of Small and Medium-Sized Enterprises: Peru, a Latin-American Emerging Market. Emerging Markets Finance and Trade, 53(1), 150-169. https://doi.org/10.1080/1540496X.2016.1156525

Perez-Bustamante, G. O., Marques, C. S. E., Jalali, M. S., \& Ferreira, F. A. F. (2016). The impact of continuous training in small and medium enterprises: Lessons from an industrial case analysis. Journal of Business Economics and Management, 17(2), 234-250. https://doi.org/10.3846/16111699. 2014.938359

Sauka, A. (2014). Measuring the competitiveness of Latvian companies. Baltic Journal of Economics, 14(1-2), 140-158. https://doi.org/10.1080/1406099X.2014.995421

Schmalensee, R. (1985). Do Markets Differ Much? The American Economic Review, 75(3), 341-351.

Schmalensee, R. (1989). Chapter 16 Inter-industry studies of structure and performance. En Handbook of Industrial Organization (Vol. 2, pp. 951-1009). Elsevier. Recuperado de http://www . sciencedirect.com/science/article/pii/S1573448X89020042

Shen, G., \& Chen, B. (2017). Zombie firms and over-capacity in Chinese manufacturing. China Economic Review, 44,327-342. https://doi.org/10.1016/j.chieco.2017.05.008

Shepherd, W. G. (1972). The Elements of Market Structure. The Review of Economics and Statistics, 54(1), 25-37. https://doi.org/10.2307/1927492

Solleiro, J. L., \& Castañón, R. (2005). Competitiveness and innovation systems: the challenges for Mexico's insertion in the global context. Technovation, 25(9), 1059-1070. https://doi.org/10.1016/ j.technovation.2004.02.005

Stojcic, N., Hashi, I., \& Telhaj, S. (2013). Restructuring and Competitiveness Empirical Evidence on Firm Behavior in New EU Member States and Candidate Countries. Eastern European Economics, 51(4), 84-107. https://doi.org/10.2753/EEE0012-8775510404

Subramanian, S., \& Reddy, V. N. (2012). Corporate governance disclosures and international competitiveness: A study of Indian firms. Asian Business 63 Management, 11(2), 195-218. https: //doi.org/10.1057/abm.2012.1

Tan, Y., Huang, Y., \& Woo, W. T. (2016). Zombie Firms and the Crowding-Out of Private Investment in China. Asian Economic Papers, 15(3), 32-55. https://doi.org/10.1162/ASEP_a_00474

Uchida, H., Miyakawa, D., Hosono, K., Ono, A., Uchino, T., \& Uesugi, I. (2015). Financial shocks, bankruptcy, and natural selection. Japan and the World Economy, 36, 123-135. https://doi.org/ $10.1016 / j \cdot$ japwor. 2015.11.002

Urionabarrenetxea, S., Garcia-Merino, J. D., San-Jose, L., \& Retolaza, J. L. (2018). Living with zombie companies: Do we know where the threat lies? European Management Journal. https://doi.org/ 10.1016/j.emj.2017.05.005

Wu, P., Gao, L., \& Gu, T. (2015). Business strategy, market competition and earnings management: Evidence from China. Chinese Management Studies, 9(3), 401-424. https://doi.org/10.1108/ CMS-12-2014-0225 